Document downloaded from:

http://hdl.handle.net/10251/147622

This paper must be cited as:

Guirao Sánchez, AJ.; Hajek, P. (2007). Schauder bases under uniform renormings. Positivity. 11(4):627-638. https://doi.org/10.1007/s11117-007-2067-9

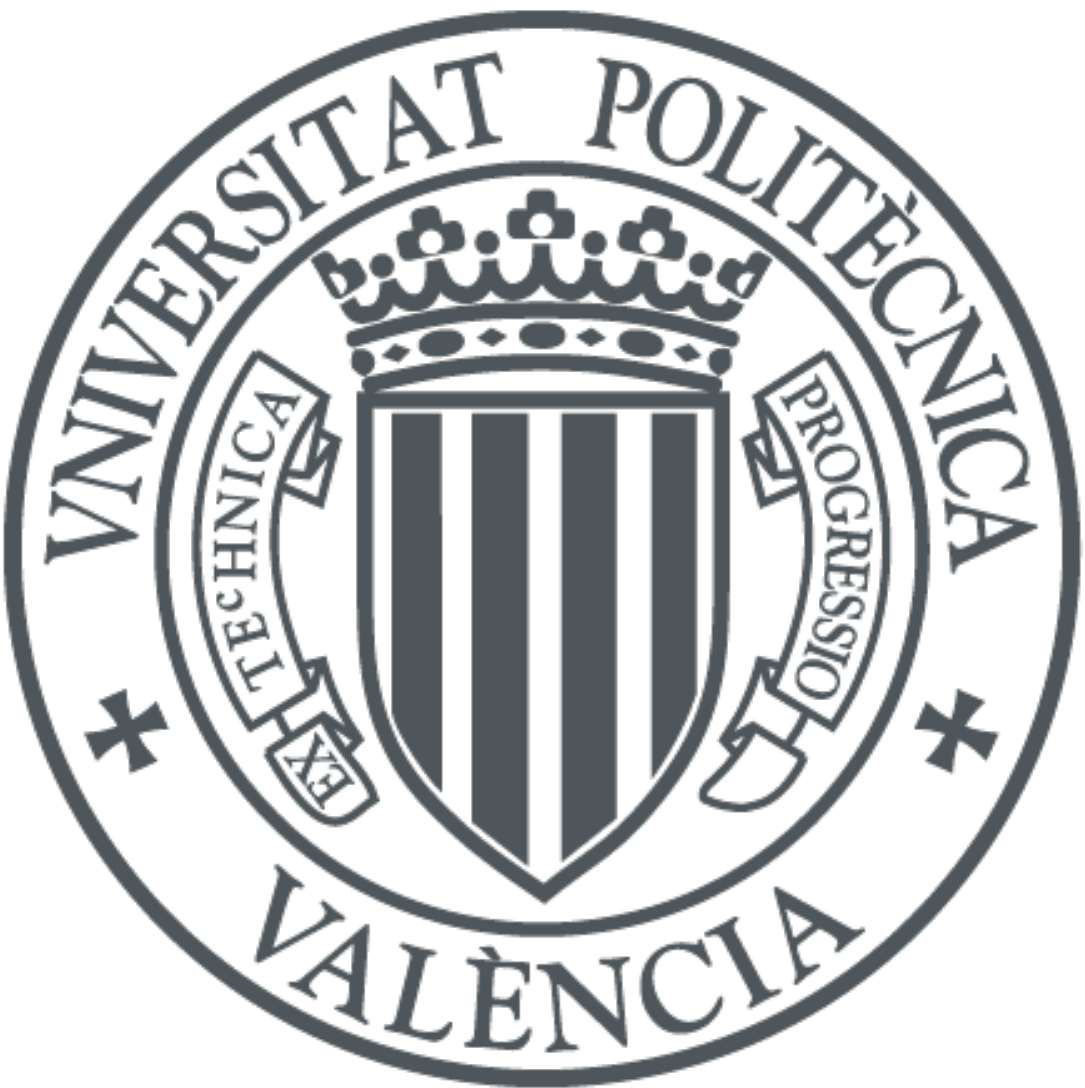

The final publication is available at

https://doi.org/10.1007/s11117-007-2067-9

Copyright Springer-Verlag

Additional Information 


\title{
SCHAUDER BASES UNDER UNIFORM RENORMINGS
}

\author{
A. J. GUIRAO AND P. HÁJEK
}

\begin{abstract}
Let $X$ be a separable superreflexive Banach space with a Schauder basis. We prove the existence of an equivalent uniformly smooth (resp. uniformly rotund) renorming under which the given basis is monotone.
\end{abstract}

\section{INTRODUCTION}

Let us start by recalling a classical notion of Schauder basis $\left\{e_{n}\right\}_{n=1}^{\infty}$ of a Banach space $X$, i.e. a sequence in $X$ such that for every $x \in X$ there is a unique sequence of coefficients $\left\{a_{n}\right\}_{n=1}^{\infty}$, such that $x=\sum a_{n} e_{n}$. A Schauder basis gives rise to the canonical sequence of finite dimensional projections $P_{n}: X \rightarrow X, P_{n}\left(\sum_{i=1}^{\infty} a_{i} e_{i}\right)=\sum_{i=1}^{n} a_{i} e_{i}$. A well-known and useful result on Schauder bases claims that $\left\{\left\|P_{n}\right\|\right\}_{n}$ is a bounded sequence ([2]). The quantity $\mathrm{bc}\left\{e_{i}\right\}=\sup _{n}\left\|P_{n}\right\|$ is called the basis constant. We say that the Schauder basis is monotone, if the value of its basis constant is 1 . The boundedness result has an equivalent reformulation in the language of renormings. Namely, a separable Banach space with a Schauder basis can be equivalently renormed so that the basis becomes monotone. The proof of this equivalence is very easy, in fact the renorming is obtained via the formula $\||| x \mid\|=\sup _{n}\left\|P_{n}(x)\right\|$. Unfortunately, from the renorming point of view, ||$|\cdot|||$ looses some subtler geometrical properties of the original norm. The question on the existence of "good" renormings, still making the given basis monotone, has received some attention in the past. For example, it is well-known that every separable Banach has an LUR renorming, in fact the collection of all equivalent LUR renormings is residual in the (metric) space of all equivalent renormings ([1]). It is therefore quite natural to expect that for every separable Banach space with a Schauder basis, there exists an equivalent LUR renorming making the basis monotone. This is indeed the case, and the proof follows along the lines of the original Kadec LUR renorming result (see [1]). Similar statements (folklore) are true also for Gateaux or uniformly Gateaux smooth case. On the

2000 Mathematics Subject Classification. 46B03.

Key words and phrases. Banach Spaces; Monotone Schauder Basis; Uniformly Fréchet Norms; Uniformly Rotund Norms.

First author supported by the grants MTM2005-08379 of MECD (Spain), 00690/PI/04 of Fundación Séneca (CARM, Spain) and AP2003-4453 of MECD (Spain), Second author supported by AV0Z10190503 and A100190502. 
other hand, quite surprisingly, the situation with Fréchet smooth renormings is different. Recall that a separable Banach space has an equivalent Fréchet smooth renorming if and only if it has a separable dual. In this case, the set of Fréchet smooth renormings (whose dual norm is LUR) is again residual among all equivalent renormings, yet we have the following theorem.

Theorem 1. Let $X$ be a separable Banach space with a separable dual (in particular having an equivalent Fréchet smooth renorming), and a Schauder basis. Then $X$ is reflexive iff for every Schauder basis of $X$ there exists some Fréchet smooth renorming of $X$ making the basis monotone.

Proof. If $X$ is reflexive, then $X^{*}$ is separable and every Schauder basis of $X$ is shrinking, so we can use the mentioned LUR result for the dual basis, in order to obtain the Fréchet smooth case for $X$ (see [1] for details on the duality of renormings). On the other hand, by Proposition 8.34 of [2], if $\left\{e_{i}\right\}$ is a monotone basis of $(X,\||\cdot|\|)$, and $\||\cdot|\| \mid$ is Fréchet smooth, then $\left\{e_{i}\right\}$ is a shrinking basis. Thus for spaces satisfying the second condition, every Schauder basis is shrinking. This condition is equivalent to reflexivity by a result of Zippin [5].

As an immediate consequence, in every non-reflexive Banach space with a separable dual and a Schauder basis ( $\operatorname{such}$ as $c_{0}$ ), there exists another Schauder basis which is not monotone under any Fréchet smooth renorming of $X$ (in spite of the rich supply of equivalent Fréchet smooth renormings for such a space).

In the present note, we settle in the positive the case of uniformly Fréchet smooth (UF) and uniformly rotund (UR) renormings, answering a question of Godefroy (which appears also explicitly in [3]), communicated to us by Zizler. We would like to thank Václav Zizler for suggesting the problem to us, as well as for some useful remarks concerning this note.

1.1. Notation and basic definitions. We start by recalling some standard notions and definitions to be used throughout this note. The standard references for most of these notions are [2], or [1].

Definition 2. Let $B$ be a bounded, closed, convex and centrally symmetric subset of a linear space $\mathbb{E}$ whose interior contains the origin. Then, we will say that $B$ is a ball of $\mathbb{E}$.

For every ball $B$ of $\mathbb{E}$, there exists a norm in $\mathbb{E}$ whose unit ball is precisely $B$. This norm is defined by the gauge functional, also known as Minkowski functional, and we will denote it, as it is usual in the literature, by $g(B, \cdot)$. We will also denote $S_{B}=\{x \in X: g(B, x)=1\}$.

Regarding differentiability, we can define, for every ball $B$ of $\mathbb{E}$, its modulus of smoothness. 
Definition 3. Let $B$ be a ball in a linear space $\mathbb{E}$. Then, its modulus of smoothness is the real function defined, for $t>0$, by

$$
\rho(B, t):=\sup \{\xi(B, x, y, t): g(B, x)=g(B, y)=1\}
$$

where

$$
\xi(B, x, y, t)=\frac{g(B, x+t y)+g(B, x-t y)-2 g(B, x)}{2} .
$$

A norm $\|\cdot\|$ in Banach space is said to be uniformly Fréchet differentiable, shortly, UF, if its modulus of smoothness $\rho$ satisfies the following condition:

$$
\lim _{t \rightarrow 0} \frac{\rho(t)}{t}=0 .
$$

Therefore we can say that, a ball $B$ of a Banach space $X$ is UF, if and only if the following condition holds:

$$
\lim _{t \rightarrow 0} \frac{\rho(B, t)}{t}=0 .
$$

Then, it is clear that $B$ will be UF if and only if its associated norm by the gauge functional, $g(B, \cdot)$, is $\mathrm{UF}$.

For every ball $B$, and $x \in \mathbb{E}$ satisfying $g(B, x)=1$, let us denote by $H(B, x)$ the union of all the support hyperplanes to $B$ at $x$. It is also useful the following modulus,

$$
\bar{\rho}(B, t):=\sup \{\xi(B, x, y, t): g(B, x)=g(B, y)=1 \text { and } y+x \in H(B, x)\} .
$$

It was shown by Figiel [4] that for each ball and for $t>0$

$$
\rho(B, t) \leq 16 \bar{\rho}(B, t) .
$$

We will refer to this result as the Figiel lemma.

\section{Preliminary Constructions}

Let $X$ be a Banach space endowed with a uniformly Fréchet differentiable norm $\|\cdot\|$, and a Schauder basis $\left\{e_{i}\right\}_{i=1}^{\infty}$. Our aim is to show that there exists an equivalent $\mathrm{UF}$ renorming of $X$ which makes $\left\{e_{i}\right\}$ a monotone Schauder basis of $X$. Therefore, we will suppose from now on that the basis constant of the Schauder basis, $b c\left\{e_{i}\right\}$ is strictly greater than 1 . Then, we can consider the norm $\|x\|_{0}=\sup \left\{\left\|P_{n}(x)\right\|: n \in \mathbb{N}\right\}$, which is known to make the basis $\left\{e_{i}\right\}$ monotone (but of course not UF, in general).

Let us denote by $B$ and $B_{0}$ the following balls:

$$
\begin{aligned}
B & :=\{x \in X:\|x\| \leq 1\}, \\
B_{0} & :=\left\{x \in X:\|x\|_{0} \leq 1\right\} .
\end{aligned}
$$

It is clear that

$$
\|\cdot\| \leq\|\cdot\|_{0} \leq b c\left\{e_{i}\right\}\|\cdot\|
$$


and therefore

$$
B_{0} \subset B \subset b c\left\{e_{i}\right\} B_{0} .
$$

Through this note $k \geq b c\left\{e_{i}\right\}$ is fixed. It is clear that the last two expressions are true replacing $b c\left\{e_{i}\right\}$ by $k$. For every natural number $n$, we will denote by $\mathbb{E}_{n}$ the range of $P_{n}$, this is, $P_{n} X=\mathbb{E}_{n}$; by $B_{n}^{\prime}$ the image $P_{n}\left(\frac{1}{k} B\right)$; and for every $y \in \mathbb{E}_{n}$, by $B_{n}^{\prime}(y)$ the set $y+B_{n}^{\prime}$. In particular $B_{n}^{\prime}(0)=B_{n}^{\prime}$ is a ball of $\mathbb{E}_{n}$ whose centre is the origin. For any $y \in \mathbb{E}_{n}$ the set $B_{n}^{\prime}(y)$ is a translate of a ball of $\mathbb{E}_{n}$. We are going to define inductively a sequence $\left\{B_{n}\right\}_{n=1}^{\infty}$ of balls of $\mathbb{E}_{n}$ as follows. Put $B_{1}:=B_{0} \cap \mathbb{E}_{1}$. Having defined $B_{n}$ for $n \geq 1$, we will define the set $B_{n+1}$ as follows: First of all let us consider the set

$$
\mathbb{B}_{n+1}:=\left\{y \in \mathbb{E}_{n+1}: P_{n}\left(B_{n+1}^{\prime}(y)\right) \subset B_{n}, \quad B_{n+1}^{\prime}(y) \cap B_{0} \neq \emptyset\right\}
$$

and then we finally set

$$
B_{n+1}:=\bigcup_{y \in \mathbb{B}_{n+1}} B_{n+1}^{\prime}(y) .
$$

We can see, for example, the construction of $B_{2}$ in figure 1.
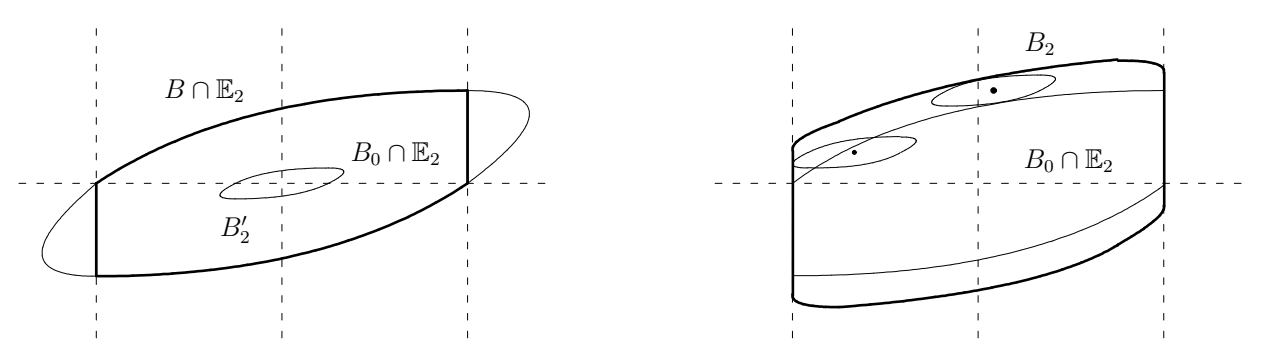

\section{Figure 1}

Lemma 4. The family of sets $\left\{B_{n}\right\}_{n \in \mathbb{N}}$ is uniformly bounded in $X$, and for every $n$, the set $B_{n}$ has non-empty interior with respect to the topology of $\mathbb{E}_{n}$.

Proof. Let us fix $n \in \mathbb{N}$ and $x \in B_{n}$. Then there exist $y, z \in \mathbb{E}_{n}$ such that $x \in B_{n}^{\prime}(y), z \in B_{0} \cap B_{n}^{\prime}(y)$ and $P_{n-1}\left(B_{n}^{\prime}(y)\right) \subset B_{n-1}$. It is clear that the vectors $x-y$ and $z-y$ lie in $B_{n}^{\prime}$. Therefore, there exist $x^{\prime}, z^{\prime} \in B$, such that $P_{n}\left(x^{\prime}\right)=k(x-y)$ and $P_{n}\left(z^{\prime}\right)=k(z-y)$, and then

$$
\begin{aligned}
\|x-y\| & =\frac{1}{k}\left\|P_{n}\left(x^{\prime}\right)\right\| \leq \frac{1}{k}\left\|P_{n}\right\|\left\|x^{\prime}\right\| \leq 1, \\
\|z-y\| & =\frac{1}{k}\left\|P_{n}\left(z^{\prime}\right)\right\| \leq \frac{1}{k}\left\|P_{n}\right\|\left\|z^{\prime}\right\| \leq 1 .
\end{aligned}
$$

Since $z \in B_{0}$ and $B_{0} \subset B$, we have $\|z\| \leq 1$. Therefore $\|x\| \leq 3$, this is $B_{n} \subset 3 B \cap \mathbb{E}_{n}$. The second part of this proof is a direct application of the following lemma, since $B \cap \mathbb{E}_{n} \subset P_{n}(B)$. 
Lemma 5. For every natural number $n$, the set $\mathbb{B}_{n}$ is non-empty. Moreover

$$
0 \in \bigcap_{n \geq 1} \mathbb{B}_{n} .
$$

Proof. It is enough to show that $0 \in \mathbb{B}_{n}$ for any $n \in \mathbb{N}$. We will prove that $0 \in \mathbb{B}_{n}$ if and only if $B_{1}^{\prime} \subset B_{1}$, which will finish the proof, because it always holds

$$
B_{1}^{\prime}=P_{1}\left(\frac{1}{k} B\right) \subset P_{1}\left(B_{0}\right) \subset B_{0} \cap \mathbb{E}_{1}=B_{1}
$$

since $B_{0}$ is a monotone ball. We will show the required equivalence by induction on $n$. For $n=2,0$ lies in $\mathbb{B}_{2}$ if and only if $P_{1}\left(B_{2}^{\prime}\right) \subset B_{1}$, since $B_{2}^{\prime}$ always intersects $B_{0}$ (the intersection contains at least the origin). But

$$
P_{1}\left(B_{2}^{\prime}\right)=P_{1}\left(P_{2}\left(\frac{1}{k} B\right)\right)=B_{1}^{\prime},
$$

which finishes the proof of this case. If the statement is true for $n-1 \geq 2$, then $0 \in \mathbb{B}_{n}$ if and only if $P_{n-1}\left(B_{n}^{\prime}\right) \subset B_{n-1}$, since the intersection between $B_{n}^{\prime}$ and $B_{0}$ is always non-empty (it contains the origin). Besides

$$
P_{n-1}\left(B_{n}^{\prime}\right)=P_{n-1}\left(P_{n}\left(\frac{1}{k} B\right)\right)=P_{n-1}\left(\frac{1}{k} B\right)=B_{n-1}^{\prime} .
$$

Therefore $0 \in \mathbb{B}_{n}$ if and only if $0 \in \mathbb{B}_{n-1}$ which is equivalent to $B_{1}^{\prime} \subset B_{1}$.

The following lemma shows that the defined sets $\left\{B_{n}\right\}_{n}$ are balls in $\mathbb{E}_{n}$, i.e., their gauge functionals are norms in $\mathbb{E}_{n}$.

Lemma 6. For every natural number $n$, the set $B_{n}$ is a ball of $\mathbb{E}_{n}$.

Proof. By lemma 4 it is clear that for every $n$, the set $B_{n}$ is bounded and contains the origin as an interior point. For the remaining properties we will use induction.

It is clear that $B_{1}$ is closed, convex and centrally symmetric. Let us suppose that $B_{n-1}$ do so for $n-1 \geq 1$.

a) We start proving that $B_{n}$ is closed. Let us consider $x \in \overline{B_{n}}$. Then there exists a sequence $\left\{x_{m}\right\}_{m}$ in $B_{n}$ converging to $x$. For every $m \in \mathbb{N}$ there exist $y_{m}$ and $z_{m}$ in $\mathbb{E}_{n}$ such that $x_{m} \in B_{n}^{\prime}\left(y_{m}\right), z_{m} \in B_{0} \cap B_{n}^{\prime}\left(y_{m}\right)$ and $P_{n-1}\left(B_{n}^{\prime}\left(y_{m}\right)\right) \subset B_{n-1}$. We can suppose that both $\left\{y_{m}\right\}$ and $\left\{z_{m}\right\}$ converge respectively to $y$ and $z$ in $\mathbb{E}_{n}$, since $B_{n}$ is bounded. We want to show that $y \in \mathbb{B}_{n}$.

Since $z_{m} \in B_{0}$ then $z \in B_{0}$, and it is clear that

$$
\begin{aligned}
& \frac{1}{k} \geq \lim _{m \rightarrow \infty}\left\|z_{m}-y_{m}\right\|=\|z-y\|, \\
& \frac{1}{k} \geq \lim _{m \rightarrow \infty}\left\|x_{m}-y_{m}\right\|=\|x-y\| .
\end{aligned}
$$


These equations mean, respectively, that $z \in B_{0} \cap B_{n}^{\prime}(y)$ and $x \in$ $B_{n}^{\prime}(y)$. Moreover, if we take $w \in B_{n}^{\prime}(y)$, then $w_{m}:=y_{m}+(w-y) \in$ $B_{n}^{\prime}\left(y_{m}\right)$ and $\left\{w_{m}\right\}_{m}$ converges to $w$. Therefore, since $P_{n-1}\left(w_{m}\right) \in B_{n-1}$ and by hypothesis $B_{n-1}$ is closed, $P_{n-1}(w) \in B_{n-1}$, this is $x \in B_{n}$.

b) Now we show that $B_{n}$ is convex. Let us take $x_{1}$ and $x_{2}$ in $B_{n}$. For $i \in$ $\{1,2\}$ there exist $y_{i} \in \mathbb{B}_{n}$ such that $x_{i} \in B_{n}^{\prime}\left(y_{i}\right)$, and $z_{i} \in B_{0} \cap B_{n}^{\prime}\left(y_{i}\right)$. Let us denote by $y=\left(y_{1}+y_{2}\right) / 2, z=\left(z_{1}+z_{2}\right) / 2$ and $x=\left(x_{1}+x_{2}\right) / 2$. Since $z_{i}-y_{i} \in B_{n}^{\prime}$ for $i \in\{1,2\}$ and $B_{n}^{\prime}$ is convex, then

$$
z=\frac{z_{1}+z_{2}}{2}=y+\frac{\left(z_{1}-y_{1}\right)+\left(z_{2}-y_{2}\right)}{2} \in B_{n}^{\prime}(y) .
$$

Therefore $z \in B_{0} \cap B_{n}^{\prime}(y)$, since $B_{0}$ is also convex. Analogously we have $x \in B_{n}^{\prime}(y)$. In order to prove that $x \in B_{n}$ we only need to see that $y \in \mathbb{B}_{n}$, so it is enough to show $P_{n-1}\left(B_{n}^{\prime}(y)\right) \subset B_{n-1}$. In fact, if $w \in B_{n}^{\prime}(y)$, then $w-y \in B_{n}^{\prime}$ and for $i \in\{1,2\}$ we have $y_{i}+(w-y) \in$ $B_{n}^{\prime}\left(y_{i}\right)$. Since $y_{i} \in \mathbb{B}_{n}$ for $i \in\{1,2\}$, then $P_{n-1}\left(y_{i}+(w-y)\right) \in B_{n-1}$ for both $i=1,2$. Therefore

$$
P_{n-1}(w)=\frac{1}{2}\left[P_{n-1}\left(y_{1}+(w-y)\right)+P_{n-1}\left(y_{2}+(w-y)\right)\right] \in B_{n-1},
$$

since, by hypothesis, $B_{n-1}$ is convex.

c) Let $x \in B_{n}$. Then there exist $y \in \mathbb{B}_{n}$ such that $x \in B_{n}^{\prime}(y)$, and $z \in B_{0} \cap B_{n}^{\prime}(y)$. It is clear that $z-y \in B_{n}^{\prime}$ and, since $B_{n}^{\prime}$ is centrally symmetric, $y-z \in B_{n}^{\prime}$. Therefore $-z=-y+(y-z) \in B_{n}^{\prime}(-y)$. But $B_{0}$ is also centrally symmetric, and thus $-z \in B_{0} \cap B_{n}^{\prime}(-y)$. Analogously we can show that $-x \in B_{n}^{\prime}(-y)$.

On the other hand, if we take $w \in B_{n}^{\prime}(-y)$, then $w+y \in B_{n}^{\prime}$, and, since $B_{n}^{\prime}$ is centrally symmetric, $-w-y \in B_{n}^{\prime}$. Therefore $-w=$ $y+(-w-y) \in B_{n}^{\prime}(y)$. Since $P_{n-1}\left(B_{n}^{\prime}(y)\right) \subset B_{n-1}$, and, by induction hypothesis, $B_{n-1}$ is centrally symmetric, $P_{n-1}(w) \in B_{n-1}$. Hence, we have shown that $P_{n-1}\left(B_{n}^{\prime}(-y)\right) \subset B_{n-1}$ and then $-y \in \mathbb{B}_{n}$. Therefore $-x \in B_{n}^{\prime}(-y) \subset B_{n}$, this is, $B_{n}$ is centrally symmetric.

In order to show that $\left\{B_{n}\right\}_{n}$ consists of UF-smooth balls, we need two auxiliary lemmas.

Lemma 7. For any $n \in \mathbb{N}$, the modulus of smoothness of $B_{n}^{\prime}$ and $B$ are related as follows:

$$
\rho\left(B_{n}^{\prime}, \cdot\right) \leq \rho(B, \cdot)
$$

Proof. Let us fix $n \in \mathbb{N}$ and $t>0$. Let us also take $x$ and $y$ such that $g\left(B_{n}^{\prime}, x\right)=g\left(B_{n}^{\prime}, y\right)=1$. In particular, since $x$ and $y$ lie on $S_{B_{n}^{\prime}}$, there exist 
$x^{\prime}, y^{\prime} \in S_{B}$ such that $P_{n}\left(x^{\prime}\right)=k x$ and $P_{n}\left(y^{\prime}\right)=k y$. Let us note that

$$
g\left(B_{n}^{\prime}, P_{n}\left(\frac{x^{\prime} \pm t y^{\prime}}{k g\left(B, x^{\prime} \pm t y^{\prime}\right)}\right)\right) \leq 1
$$

This inequality is equivalent to $g\left(B_{n}^{\prime}, x \pm t y\right) \leq g\left(B, x^{\prime} \pm t y^{\prime}\right)$. Therefore

$$
\xi\left(B_{n}^{\prime}, x, y, t\right) \leq \xi\left(B, x^{\prime}, y^{\prime}, t\right) \leq \rho(B, t),
$$

and taking suprema over $(x, y) \in S_{B_{n}^{\prime}} \times S_{B_{n}^{\prime}}$ we have the required result.

Lemma 8. Let $A$ and $B$ be two balls in a linear space $\mathbb{E}$. Let $x \in \mathbb{E}$ such that $x+B \subset A$. Then, for any $z \in \mathbb{E}$ the following inequality holds:

$$
g(A, z) \leq g(B, z-x)+|1-g(B, z-x)| g(A, x) .
$$

Proof. Let us note that the vector

$$
\widetilde{z}=x+\frac{z-x}{g(B, z-x)}
$$

lies in the set $A$, see figure 2 . Then, it is clear that

$$
\left.\tilde{z}=x+\frac{z-x}{g(B, z-x)}\right) \text {. }
$$

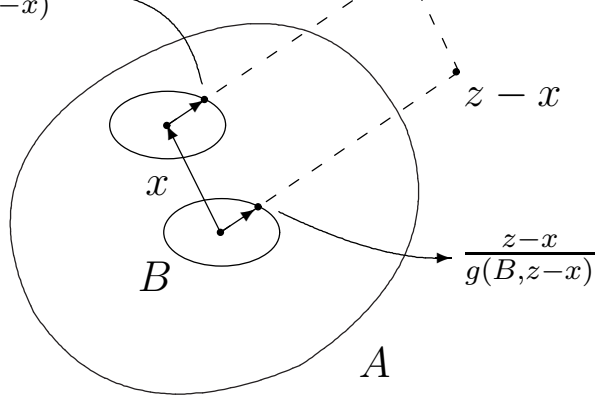

FiguRE 2

Therefore,

$$
\begin{aligned}
g(B, z-x) & \geq g(A, z-x(1-g(B, z-x))) \\
& \geq g(A, z)-g(A, x(1-g(B, z-x))) .
\end{aligned}
$$

Thus we finally obtain

$$
\begin{aligned}
g(A, z) & \leq g(B, z-x)+g(A, x(1-g(B, z-x))) \\
& =g(B, z-x)+|1-g(B, z-x)| g(A, x) .
\end{aligned}
$$

which concludes the proof. 
Proposition 9. For every $n \in \mathbb{N}$ the modulus of smoothness of $B_{n}$ satisfies

$$
\rho\left(B_{n}, t\right) \leq 32 \rho(B, 3 k t) \text {. }
$$

Proof. Let us fix $n \in \mathbb{N}$, and $t>0$. Let us also consider $x$ and $h$ points on $S_{B_{n}}$. By construction, there exists $y \in \mathbb{B}_{n}$ such that $g\left(B_{n}^{\prime}, x-y\right)=1$. Applying lemma 8 to $B_{n}, B_{n}^{\prime}$ and the point $y$, we can assure that

$$
g\left(B_{n}, x \pm t h\right) \leq g\left(B_{n}^{\prime}, x \pm t h-y\right)+\left[g\left(B_{n}^{\prime}, x \pm t h-y\right)-1\right] g\left(B_{n}, y\right)
$$

under the hypothesis $g\left(B_{n}^{\prime}, x \pm t h-y\right) \geq 1$. Therefore,

$$
\begin{aligned}
\xi\left(B_{n}, x, h, t\right) & \leq \xi\left(B_{n}^{\prime}, x-y, h, t\right)+g\left(B_{n}, y\right) \xi\left(B_{n}^{\prime}, x-y, h, t\right) \\
& =\left(1+g\left(B_{n}, y\right)\right) \xi\left(B_{n}^{\prime}, x-y, h, t\right) \leq 2 \xi\left(B_{n}^{\prime}, x-y, h, t\right)
\end{aligned}
$$

If we denote by $\vartheta=g\left(B_{n}^{\prime}, h\right)$, we have

$$
\xi\left(B_{n}, x, h, t\right) \leq 2 \xi\left(B_{n}^{\prime}, x-y, h, t\right)=2 \xi\left(B_{n}^{\prime}, x-y, \vartheta^{-1} h, \vartheta t\right) \leq 2 \rho\left(B_{n}^{\prime}, \vartheta t\right) .
$$

Since $\frac{1}{k} B \cap \mathbb{E}_{n} \subset B_{n}^{\prime}$, by the monotonicity of the gauge functional we have

$$
\begin{aligned}
\vartheta & =g\left(B_{n}^{\prime}, h\right) \leq g\left(\frac{1}{k} B \cap \mathbb{E}_{n}, h\right)=k g\left(B \cap \mathbb{E}_{n}, h\right) \\
& =k g(B, h)=k\|h\| .
\end{aligned}
$$

But, since $h \in B_{n}$, using lemma 4 , we obtain $\|h\| \leq 3$.

Since the modulus of smoothness is a non-decreasing function, we finally have

$$
\xi\left(B_{n}, x, h, t\right) \leq 2 \rho\left(B_{n}^{\prime}, \vartheta t\right) \leq 2 \rho\left(B_{n}^{\prime}, 3 k t\right) \leq 2 \rho(B, 3 k t) .
$$

The last inequality follows directly from lemma 7. Taking suprema over $x$ and $h$ satisfying $g\left(B_{n}^{\prime}, x \pm t h-y\right) \geq 1$ we obtain that

$$
\bar{\rho}\left(B_{n}, t\right) \leq 2 \rho(B, 3 k t) .
$$

The proof finishes applying Figiel's lemma.

\section{Uniformly Fréchet and Monotone Renorming}

Now, we are ready to construct the new norm in $X$ which will satisfy both uniformly Fréchet differentiability and monotonicity. In order to get it let us consider first some new sets. Let us fix $m \in \mathbb{N}$ and define

$$
\widetilde{B}_{m}:=\bigcap_{n \geq m}\left(B_{n} \cap \mathbb{E}_{m}\right)=\left(\bigcap_{n \geq m} B_{n}\right) \cap \mathbb{E}_{m} .
$$

We need to check if these new sets define, in fact, new norms in $\mathbb{E}_{m}$ for each natural $m$, respectively. 
Lemma 10. The family of sets $\left\{\widetilde{B}_{m}\right\}_{m \in \mathbb{N}}$ is uniformly bounded in $X$, and for every fixed $m$, the set $\widetilde{B}_{m}$, with respect to the topology of $\mathbb{E}_{n}$, has non-empty interior. Moreover

$$
\frac{1}{k} B \cap \mathbb{E}_{m} \subset \widetilde{B}_{m} \subset 3 B
$$

Proof. Using the proof of lemma 4 it is clear that

$$
\widetilde{B}_{m}=\bigcap_{n \geq m}\left(B_{n} \cap \mathbb{E}_{m}\right) \subset \bigcap_{n \geq m}\left(\left(3 B \cap \mathbb{E}_{n}\right) \cap \mathbb{E}_{m}\right)=3 B \cap \mathbb{E}_{m} \subset 3 B .
$$

On the other hand, by lemma 5 we know that

$$
\widetilde{B}_{m}=\bigcap_{n \geq m}\left(B_{n} \cap \mathbb{E}_{m}\right) \supset \bigcap_{n \geq m}\left(\left(\frac{1}{k} B \cap \mathbb{E}_{n}\right) \cap \mathbb{E}_{m}\right)=\frac{1}{k} B \cap \mathbb{E}_{m} .
$$

Lemma 11. For any natural number $m$, the set $\widetilde{B}_{m}$ is a ball in $\mathbb{E}_{m}$.

Proof. By the previous lemma, these sets are bounded and have the origin as an interior point. It remains to show that these sets are closed, convex and centrally symmetric. However, these three properties are evident, because each $\widetilde{B}_{m}$ is the intersection of sets that are closed, convex and centrally symmetric.

Proposition 12. For any natural number $m$, the modulus of smoothness of $\widetilde{B}_{m}$ satisfies

$$
\rho\left(\widetilde{B}_{m}, t\right) \leq 32 \rho(B, 3 k t)
$$

Proof. For a fixed $m \in \mathbb{N}$, we consider $n \geq m$ and take $x \in B_{n+1} \cap \mathbb{E}_{m}$. Since $P_{n}(x) \in B_{n}$, we have that $x=P_{m}(x)=P_{n}(x) \in B_{n}$. This is, the sequence $\left\{B_{n} \cap \mathbb{E}_{m}\right\}_{n \geq m}$ is decreasing. Therefore it converges in the Hausdorff metric of $\mathbb{E}_{m}$ to its intersection, this is, to $\widetilde{B}_{m}$. It is then well-known, and standard to prove, that

$$
\lim _{n \rightarrow \infty} g\left(B_{n} \cap \mathbb{E}_{m}, \cdot\right)=g\left(\widetilde{B}_{m}, \cdot\right),
$$

where the limit is understood as uniform convergence on bounded sets in $\mathbb{E}_{m}$.

Let us take $x$ and $y$ such that $g\left(\widetilde{B}_{m}, x\right)=g\left(\widetilde{B}_{m}, y\right)=1$. We have

$$
\begin{aligned}
\xi\left(B_{n}, x, y, t\right) & =g\left(B_{n}, x\right) \xi\left(B_{n}, \frac{x}{g\left(B_{n}, x\right)}, \frac{y}{g\left(B_{n}, y\right)}, t \frac{g\left(B_{n}, y\right)}{g\left(B_{n}, x\right)}\right) \\
& \leq g\left(B_{n}, x\right) \rho\left(B_{n}, t \frac{g\left(B_{n}, y\right)}{g\left(B_{n}, x\right)}\right) .
\end{aligned}
$$


Then by proposition 9

$$
\xi\left(B_{n}, x, y, t\right) \leq g\left(B_{n}, x\right) 32 \rho\left(B, 3 k t \frac{g\left(B_{n}, y\right)}{g\left(B_{n}, x\right)}\right),
$$

and taking limits, using equation (3.1) and the continuity of $\rho(B, \cdot)$, we have

$$
\xi\left(\widetilde{B}_{m}, x, y, t\right) \leq 32 \rho(B, 3 k t) .
$$

Taking suprema over $x$ and $y$ we obtain $\rho\left(\widetilde{B}_{m}, t\right) \leq 32 \rho(B, 3 k t)$ as we wanted to show.

Finally, let us define the set

$$
\widetilde{B}:=\varlimsup_{\bigcup_{m \in \mathbb{N}} \widetilde{B}_{m}}{ }^{\|\cdot\|} .
$$

Lemma 13. For any natural number $m$, the following conditions hold:

(i) For every $n>m, \widetilde{B}_{n} \cap \mathbb{E}_{m}=\widetilde{B}_{m}$.

(ii) $\widetilde{B}_{n} \subset \widetilde{B}_{m}+\operatorname{span}\left\{e_{j}: m<j \leq n\right\}$.

(iii) $\widetilde{B}_{m}=\widetilde{B} \cap \mathbb{E}_{m}$.

Proof. (i) Since the sequence $\left\{B_{r} \cap \mathbb{E}_{m}\right\}_{r \geq m}$ is decreasing, then

$$
\widetilde{B}_{n} \cap \mathbb{E}_{m}=\bigcap_{r \geq n}\left(B_{r} \cap \mathbb{E}_{n}\right) \cap \mathbb{E}_{m}=\bigcap_{r \geq n}\left(B_{r} \cap \mathbb{E}_{m}\right)=\bigcap_{r \geq m}\left(B_{r} \cap \mathbb{E}_{m}\right)=\widetilde{B}_{m} .
$$

(ii) It is enough to show that $\widetilde{B}_{n} \subset \widetilde{B}_{n-1}+\operatorname{span}\left\{e_{n}\right\}$. Let us take $x \in \widetilde{B}_{n}$. Then $x \in B_{r} \cap \mathbb{E}_{n}$ for every $r \geq n$. Let us consider $r \geq n-1$. Then $x \in B_{r+1} \cap \mathbb{E}_{n}$ and therefore $x$ lies in $\left(B_{r}+\operatorname{span}\left\{e_{r+1}\right\}\right) \cap \mathbb{E}_{n}$. This implies that $P_{n-1}(x) \in B_{r} \cap \mathbb{E}_{n-1}$. Since $r \geq n-1$ is arbitrary, we have that $P_{n-1}(x)$ lies in $\widetilde{B}_{n-1}$, this is, $x \in \widetilde{B}_{n-1}+\operatorname{span}\left\{e_{n}\right\}$.

(iii) Clearly $\widetilde{B}_{m} \subset \widetilde{B} \cap \mathbb{E}_{m}$. On the other hand, let us take $x \in \widetilde{B} \cap \mathbb{E}_{m}$. Then, there exists a sequence $\left\{x_{n}\right\}_{n}$ which converges to $x$ and such that $x_{n} \in \widetilde{B}_{m_{n}}$ for certain values $m_{n} \in \mathbb{N}$. Without loss of generality, we can reduce our proof to two cases:

- If $m_{n} \leq m$ for every $n \in \mathbb{N}$, then by the first item of this lemma, $x_{n} \in \widetilde{B}_{m}$ and, since $\widetilde{B}_{m}$ is closed, $x \in \widetilde{B}_{m}$.

- If $m_{n}>m$ for every $n \in \mathbb{N}$, by the second item of this lemma we have $P_{m}\left(x_{n}\right) \in \widetilde{B}_{m}$. But, since $x \in \mathbb{E}_{m}$, we have that $\left\{P_{m}\left(x_{n}\right)\right\}_{n}$ converges to $x$, and therefore $x \in \widetilde{B}_{m}$.

The following proposition summarizes the main properties of $\widetilde{B}$. Condition (i) claims that it is an equivalent ball in $X$, (ii) implies that the basis $\left\{e_{i}\right\}$ is monotone with respect to the associated norm of $\widetilde{B}$, and (iii) gives an estimate of the modulus of uniform smoothness. 
Proposition 14. $\widetilde{B}$ is a ball of $X$, which satisfies the following conditions:

(i) $\widetilde{B} \subset 3 B \subset 3 k \widetilde{B}$.

(ii) For every $x \in \widetilde{B}$ and for every natural number $n, P_{n}(x) \in \widetilde{B}$.

(iii) $\rho(\widetilde{B}, t) \leq 32 \rho(B, 3 k t)$.

Proof. By lemma 13 (iii), the sequence $\left\{\widetilde{B}_{m}\right\}_{m}$ is increasing. Therefore $\widetilde{B}$ is clearly convex and centrally symmetric. It follows from the definition of $\widetilde{B}$ that it is closed in $X$. In order to prove that $\widetilde{B}$ is a ball we just need to show that it contains the origin as an interior point, which is a consequence of item (i). Therefore we only need to show (i),(ii) and (iii).

(i) By lemma 10 we have that $\widetilde{B}_{m} \subset 3 B$ for every $m \in \mathbb{N}$, therefore it is clear that $\widetilde{B} \subset 3 B$. On the other hand, let us take $x$ such that $\|x\|<1 / k$. Since the sequence $\left\{P_{m}(x)\right\}_{m}$ converges to $x$, there exists $m_{0}$, such that if $m \geq m_{0}$ then $\left\|P_{m}(x)\right\| \leq 1 / k$. Since, by lemma 10 , $B \cap \mathbb{E}_{m} \subset k \widetilde{B}_{m}$, we have $P_{m}(x) \in \widetilde{B}_{m}$ for every $m \geq m_{0}$, and then $P_{m}(x) \in \widetilde{B}$ for every $m \geq m_{0}$. Therefore $x$ lies in $\widetilde{B}$. Then $s B \subset \widetilde{B}$ for any $s<1 / k$. Taking closures $B \subset k \widetilde{B}$.

(ii) Let us take $x \in \widetilde{B}$ and $m \in \mathbb{N}$. Let us also take $\left\{x_{n}\right\} \subset \widetilde{B}$ in such a way $x_{n} \in \widetilde{B}_{m_{n}}$. Without loss of generality, we can reduce our proof to two cases:

- If $m_{n} \leq m$ for every $n \in \mathbb{N}$, then, by lemma 13 (i), $x_{n} \in \widetilde{B}_{m}$, and then $P_{m}(x)=x$ lies in $\widetilde{B}_{m} \subset \widetilde{B}$.

- If $m_{n}>m$ for every $n \in \mathbb{N}$, then, by lemma 13 (ii), $P_{m}\left(x_{n}\right) \in \widetilde{B}_{m}$, thus $P_{m}(x)$ lies in $\widetilde{B}_{m} \subset \widetilde{B}$.

(iii) From the previous results it is clear that the restriction of $g(\widetilde{B},$.$) to$ $\mathbb{E}_{m}$ coincides with $g\left(\widetilde{B}_{m}, \cdot\right)$. Let us fix $t>0$ and consider $x$ and $y$ such that $g(\widetilde{B}, x)=g(\widetilde{B}, y)=1$. Let us denote $x_{m}=P_{m}(x), y_{m}=P_{m}(y)$, $\theta_{m}=g\left(\widetilde{B}_{m}, x_{m}\right)$ and $\vartheta_{m}=g\left(\widetilde{B}_{m}, y_{m}\right)$. Then, by proposition 12 ,

$$
\begin{aligned}
\xi\left(\widetilde{B}, x_{m}, y_{m}, t\right) & =\xi\left(\widetilde{B}_{m}, x_{m}, y_{m}, t\right)=\theta_{m} \xi\left(\widetilde{B}_{m}, \frac{x_{m}}{\theta_{m}}, \frac{y_{m}}{\vartheta_{m}}, t \frac{\vartheta_{m}}{\theta_{m}}\right) \\
& \leq \theta_{m} \rho\left(\widetilde{B}_{m}, t \frac{\vartheta_{m}}{\theta_{m}}\right) \leq \theta_{m} 32 \rho\left(B, 3 k t \frac{\vartheta_{m}}{\theta_{m}}\right) .
\end{aligned}
$$

Therefore

$$
\begin{aligned}
\xi(\widetilde{B}, x, y, t) & =\lim _{m \rightarrow \infty} \xi\left(\widetilde{B}, x_{m}, y_{m}, t\right) \leq \lim _{m \rightarrow \infty} \theta_{m} 32 \rho\left(B, 3 k t \frac{\vartheta_{m}}{\theta_{m}}\right) \\
& =32 \rho(B, 3 k t) .
\end{aligned}
$$

Taking suprema over $x$ and $y$ leads to the required conclusion. 
As a simple corollary of this proposition we obtain the main results of our note.

Theorem 15. Let $X$ be a Banach space with a Schauder basis $\left\{e_{i}\right\}_{i=1}^{\infty}$ and a UF-norm $\|\cdot\|$. Then there exists an equivalent UF renorming $\| \cdot|\cdot| \mid$ such that $\left\{e_{i}\right\}$ is a monotone Schauder basis of $(X,|\| \cdot|||)$. Moreover, the moduli of uniform smoothness of $\|\cdot\|$ and $\||\cdot|\|$ are equivalent (in the sense of [4]).

Dualizing, we have the following result.

Theorem 16. Let $X$ be a separable superreflexive Banach space with a Schauder basis. Then there exists an equivalent UR renorming under which the basis is monotone.

Proof. As $X$ is superreflexive, by a well-known result of Enflo [6], there exist a UR-norm $\|\cdot\|$ on $X$. Since the basis is also shrinking, $\left(X^{*},\|\cdot\|\right)$ is UF and $\left\{e_{i}^{*}\right\}$ is its Schauder basis. We can apply Theorem 15 to $X^{*}$, to obtain an equivalent UF-norm in $X^{*}$ such that the basis $\left\{e_{i}^{*}\right\}$ is monotone. By duality, this means that $X^{* *}=X$ has an equivalent UR-norm making the basis $\left\{e_{i}\right\}$ monotone.

\section{REFERENCES}

[1] R. Deville, G. Godefroy, V. Zizler, Smoothness and renormings in Banach spaces, Pitman Monographs and Surveys 64, Longman Ed (1993).

[2] M. Fabian, P. Habala, P. Hájek, V. Montesinos, J. Pelant and V. Zizler, Functional analysis and infinite dimensional geometry, Canadian Math. Soc. Books, Springer Verlag, (2001).

[3] M. Fabian, V. Montesinos and V. Zizler, Smoothness in Banach spaces. Selected problems, Rev. R. Acad. Cien. Serie A Mat. 100, (2006), 101-125.

[4] T. Figiel, On the moduli of convexity and smoothness, Studia Math. 56, (1976), 121155.

[5] M. Zippin, A remark on bases and reflexivity in Banach spaces, Isr. J. Math. 6, (1968), 74-79.

[6] P. Enflo, Banach spaces which can be given an equivalent uniformly convex norm, Isr. J. Math 13, (1972), 281-288.

Departamento de Matemáticas, Universidad de Murcia, 30100 Espinardo (MurCia), SPAIN

Mathematical Institute, AV ČR, Žitná 25, 11567 Praha 1, Czech Republic

E-mail address: ajguirao@um.es and hajek@math.cas.cz 